\title{
Analysis of Cavitation Instabilities in a Four-Blade Inducer
}

\author{
O. Coutier-Delgosha, A. Dazin, G. Caignaert, and G. Bois \\ Arts et Metiers ParisTech/LML Laboratory, 8 boulevard Louis XIV, 59046 Lille Cedex, France \\ Correspondence should be addressed to O. Coutier-Delgosha, olivier.coutier@lille.ensam.fr
}

Received 26 December 2011; Accepted 18 April 2012

Academic Editor: Meinhard Taher Schobeiri

Copyright ( 12012 O. Coutier-Delgosha et al. This is an open access article distributed under the Creative Commons Attribution License, which permits unrestricted use, distribution, and reproduction in any medium, provided the original work is properly cited.

\begin{abstract}
The cavitating behavior of a four-blade inducer tested in the LML laboratory large test facility is considered in the present paper. Experimental investigations based on unsteady pressure measurements and records from a six-component balance mounted on the inducer shaft are performed. Spectral analysis of the signals enables to detect several characteristic frequencies related to unbalanced two-phase flow patterns. The objective of the present paper is the understanding of the physical phenomena associated to these frequencies. Therefore, wavelet decomposition, flow visualizations, and direct analysis of the high-frequency force, moment, and pressure signals are applied. Results at nominal flow rate only are considered. Not only classical unbalanced cavitation patterns, but also unexpected flow organizations are discussed.
\end{abstract}

\section{Introduction}

Rocket engine turbopump inducers are designed to operate in cavitating conditions, because of the low pressure of LH2 and LOX in the tanks. However, development of cavitation must be controlled to avoid any reduction of the inducer performance. Attention is also focused on instabilities associated with cavitation, since they are responsible for unbalanced flow pattern that compromise the rotor equilibrium. Selfoscillation of the sheet cavities on the blades may also generate problematic pressure fluctuations at the inducer outlet.

The understanding of these different unsteady flow behaviors has not been completed, yet, although significant progress has been achieved in the last decades. Experimental investigations of the behavior of various inducers, characterized by a blade number varying usually between 2 and 5, have been conducted for example in Pisa University (Italy) in collaboration with Avio, in Arts et Metiers ParisTech and CREMHyG (France) in collaboration with SNECMA Moteurs, and in Osaka University (Japan) by Tsujimoto and colleagues in cooperation with the JAXA program. In these different studies, several recurrent unstable behaviors have been identified: (i) rotating cavitation is characterized by a nonsymmetrical two-phase flow pattern, which rotates faster or slower than the inducer, leading to so-called super-synchronous or subsynchronous regimes, respectively. Rotation speed in the inducer rotating frame is generally low, leading in the absolute frame to frequencies close to the inducer rotation one. Such phenomenon, which leads to significant radial forces, has been detected in nearly all tested inducers [1-3] (Tsujimoto et al. 1997). (ii) A surge mode oscillation characterized by a global pulsation of the vapor areas at low frequency: such oscillations have been investigated in simpler configurations of 2D Venturi profiles or $2 \mathrm{D}$ foil sections in cavitation tunnels and analyzed in details by many authors [4-7]. (iii) Rotating stall in backflow vortices: such behavior has been identified by Tsujimoto and colleagues [8] in the case of cavitation clouds extending upstream from the inducer inlet. It basically consists in a slow rotation of this detached cavitation, at frequency that depends on the number of vortices.

The occurrence of these different flow patterns strongly depends on the cavitation development in the machine. A typical sketch in the case of a four-blade inducer is given for example in Figure 1.

At cavitation inception, only a steady and balanced flow pattern with one short attached cavity on each blade is observed from flow visualizations. When cavitation parameter is slightly decreased, a steady and alternate cavitating 


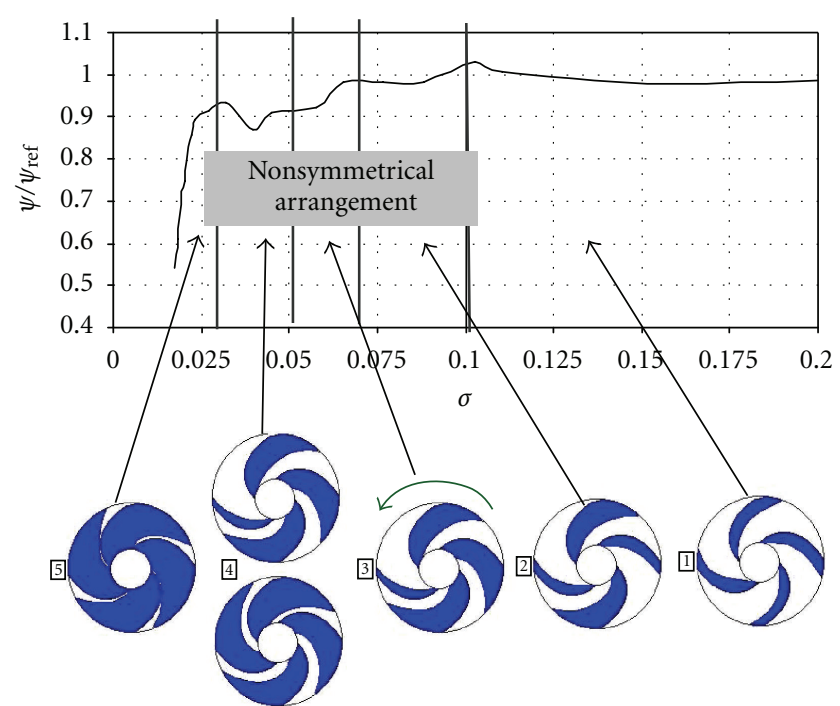

FIGURE 1: Sketches of cavitation patterns and performance evolution as the cavitation number decreases in a four-blade inducer [9].

configuration appears with alternatively one short and one long cavity. For a lower cavitation parameter, just above breakdown, rotating cavitation can be identified: unbalanced attached cavities are observed in the different channels, their distribution rotating faster than the inducer. Finally near the breakdown of the inducer, a steady and balanced flow pattern with fully developed cavitation is observed.

More recently, supplementary unstable flow patterns have been identified in particular configurations: (i) higherorder rotating cavitation has been reported first by Fujii et al. [10], from Osaka University, in the case of a threeblade inducer. Frequency related to this phenomenon is 5 $f_{\text {ref }}$ in the absolute frame. (ii) Higher-order cavitation surge: this axial instability has been found by the Osaka Team at frequency $5 f_{\text {ref }}$, and also by researchers from Pisa University at frequencies $4.4 f_{\text {ref }}$ and $6.6 f_{\text {ref }}$ in the case of a two-blade inducer. In both cases, the phenomenon was detected at high flow coefficient.

These instabilities induce some strong radial forces that may perturb the rotor balance, and important pressure fluctuations in the lines. They must be quantified and controlled to avoid any major effect on the global pump behavior. Their detection is usually based, in all aforementioned references, on pressure transducers mounted on the inducer shroud at several angles in the same section. The analysis of the phase difference between the transducers enables to distinguish axial instabilities (characterized by zero-phase difference) from rotating cavitation (where a phase difference equal to the angle difference is obtained).

As can be seen, the physical characterization of the flow instabilities related to cavitation is still weak, which explains that only a few attempts have been performed to explain inception and mechanisms of rotating cavitation [11, 12]. Yoshida and colleagues state that inception of unstable behaviours is mainly governed by the sheet cavity length $L_{c}$. Sheet cavities longer than the cascade throat remain equal

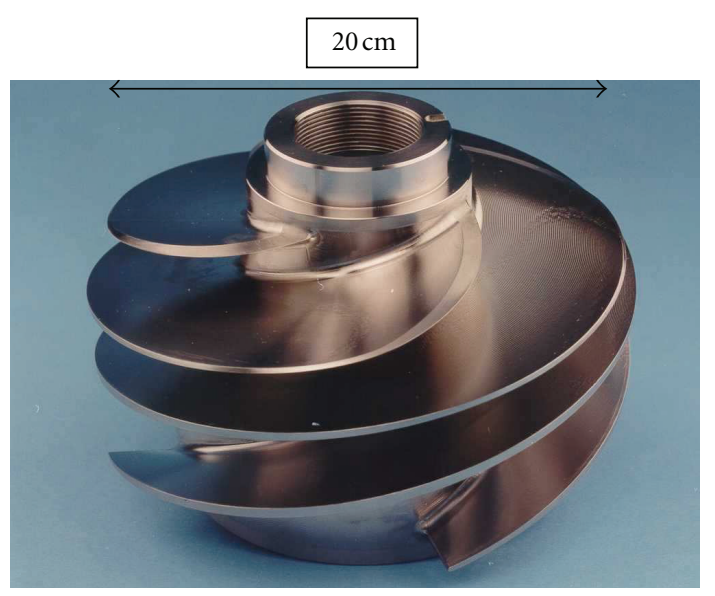

FIGURE 2: Inducer geometry.

because they are fully constrained by the adjacent blade. In opposition, sheet cavities that do not reach the cascade throat $\left(0.8<L_{c} / h<1\right)$ are characterized by a degree of freedom, which may lead to unstable cavitation such as synchronous nonsymmetrical flow patterns. However, no consensus is presently obtained concerning too the mechanisms that are responsible for the inception of unbalanced flow patterns as the ones that control the successive synchronous and nonsynchronous regimes that are usually observed in inducers.

Conversely, several studies have been conducted to investigate the effects of the inducer design on its unsteady behavior: it has been found, for example, that the shape of the hub [13], of the leading edge [14], (Bakir et al. 2002), and even of the casing [15] have significant influence on cavitation instabilities. The effects of unequal spacing of the blades have been also investigated by numerical simulations [16]. All inducers have been usually manufactured with one to 4 blades in the inlet section.

In the present paper, the cavitating behaviour of a fourblade inducer (Figure 2) is investigated. Experiments have been performed in the large test facility of ENSAM Lille, with the collaboration of the CNES (French Space Agency) and SNECMA Moteurs. Although a large range of mass flow rates, inlet pressures, and rotation speeds have been tested, attention is focused presently on a few hydrodynamic conditions only, at nominal mass flow rate $Q_{n}$ and reference rotation speed $N_{\text {ref }}$. The successive flow instabilities, which are encountered when the inlet pressure is progressively decreased, are analysed in details, in order to improve their characterization and physical understanding. Original force measurements performed with a balance mounted on the inducer shaft, associated with unsteady pressure measurements on the inducer shroud, enable to obtain data both in the absolute and inducer rotating frame, which enriches the analysis of the results.

Note that all results presented in the paper are dimensionless for reasons of confidentiality. So, the "+" indicates that each variable $V^{+}$is $V / V_{0}$ where $V_{0}$ is a reference value which is not given. 


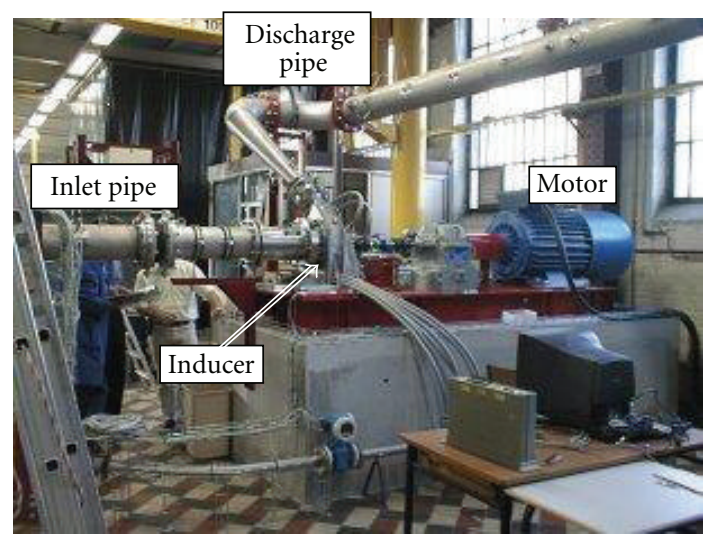

FIgURE 3: General view of the LML large test facility.

\section{Experimental Setup}

2.1. Test Facility. The LML laboratory large test facility devoted to the study of axial pumps in cavitating conditions has been used for the experiments. This two-stairs facility is equipped with a $200 \mathrm{~kW}$ motor that enables to reach an inducer rotation speed of $6000 \mathrm{rpm}$. The device upstairs (Figure 3) is mainly composed of the inducer to be tested and a stator downstream from the inducer that conducts the flow towards the discharge pipe. The inlet and discharge pipes are connected to two tanks. A free surface is maintained in the upstream one, so that the pressure can be controlled in the installation, from about 0.1 bars up to 16 bars. A part $Q_{s}$ of the volume flow rate is taken at the inducer outlet for the axial equilibrium device, and then reinjected in the upstream tank. Downstairs are located two large resorption tanks used for the extraction of dissolved gas, a variable head loss device that enables to control the flow rate, and the vacuum pump used to decrease the pressure in the inlet tank. A heat exchanger is also connected to the facility to control the water temperature.

2.2. Acquisition Device. The inducer test section is equipped with a large variety of sensors devoted either to the characterization of steady flow properties, or to the analysis of the flow unsteady fluctuations.

Concerning steady flow properties, the torque $M_{x}$ and the rotation speed $N$ are measured with a Torquemaster TM213 torque meter, while a Rosemount AP6 pressure transducer (range 0-6 bars) is used for the inlet pressure $P_{i}$. A Rosemount DP7E22 differential pressure transducer (range 0-16 bars) connected between $P_{i}$ and $P_{d}$ is used to obtain directly the elevation $\Delta P$. The main flow rate $Q_{m}$ and the recirculating one $Q_{s}$ are measured with Endress + Hauser electromagnetic flow meters. The motor shaft rotation speed is also controlled with a photoelectric cell.

Temperature measurements are performed on the upstream and downstream bearings of the inducer shaft, to control their increase at high rotation speed, and the water temperature is measured to regulate the heat exchanger operation so that a $25^{\circ} \mathrm{C}$ temperature is maintained in the flow. The torque $M_{x}$ and the rotation speed $N$ are acquired at frequency $100 \mathrm{~Hz}$ during $10 \mathrm{~s}$, while all other signals are recorded at frequency $40 \mathrm{~Hz}$ during $140 \mathrm{~s}$. From these records, mean and RMS values of the signals are calculated for each investigated flow condition. Note that the inlet and outlet pressures are measured in the inlet and delivery pipes more than one meter upstream and downstream from the inducer, so the calculated pressure head includes the head losses between the two pressure taps, including the one in the stator and in the downstream $90^{\circ}$ bends that can be seen in Figure 4.

Absolute uncertainties on the mean flow characteristics are $0.5 \%$ of $Q_{\text {ref }}$ for $Q_{m}, 0.05 \%$ of $Q_{\text {ref }}$ for $Q_{s}, 0.25 \%$ of $N_{\text {ref }}$ for $N, 2 \%$ of the noncavitating torque value for $M_{x}$, and $0.2 \%$ of the measurement range for the upstream pressure and $\Delta P$. Precision regarding the cavitation number and the head, torque, and flow rate coefficients depend on the flow conditions and will be indicated in the figures hereafter.

Unsteady flow properties are investigated with nine Kistler 701A piezo-electric pressure transducers (Figure 4): six of them are located in the upstream and delivery pipes, two other ones $\left(P_{1}^{\prime}\right.$ and $\left.P_{2}^{\prime}\right)$ are located at the inlet of the inducer test section, and the last one $P_{3}^{\prime}$ is between the inducer and the stator. All transducers are mounted flush to the internal pipe wall. Four accelerometers are also installed on the inducer casing to measure the vibrations in the axial and radial directions. A six-component balance is mounted on the shaft to obtain the axial force $F_{x}$, the radial forces $F_{y}$ and $F_{z}$, the torque $M_{x}$, and also the bending moments $M_{y}$ and $M_{z}$. These 19 parameters are recorded simultaneously at frequency $2048 \mathrm{~Hz}$ with the LMS CADA-X code. An antialiasing filtering (Butterworth, $800 \mathrm{~Hz},-50 \mathrm{~dB} /$ octave) is applied during acquisition.

Relative uncertainty on the pressure and vibration measurements is found to be close to $1 \%$ in noncavitating conditions and $2 \%$ in cavitating conditions. Precision on the forces and moments measured by the balance is estimated to $1 \%$ of the measurement ranges.

2.3. Experimental Process. Unsteady flow properties are investigated by varying continuously the inlet pressure from 2 bars down to the $25 \%$ drop of the inducer head. The duration of the pressure decrease is about $360 \mathrm{~s}$, which may result in quasisteady flow conditions (this point is checked in [17]). During this process, the variable head loss device position is not modified, so the mass flow rate progressively decreases together with the inducer elevation. The present study focuses on the results obtained at nominal mass flow rate $Q_{n}$ and reference rotation speed $N_{\text {ref }}$. However, data recorded at other rotation speeds are also used, because (i) visualizations have been performed at $0.6 N_{\text {ref }}$ and (ii) intensity of some fluctuations decreases with $N$, so they can be detected only at high values of $N$.

\section{Methodology}

Three methods have been applied for the analysis of the instabilities. 


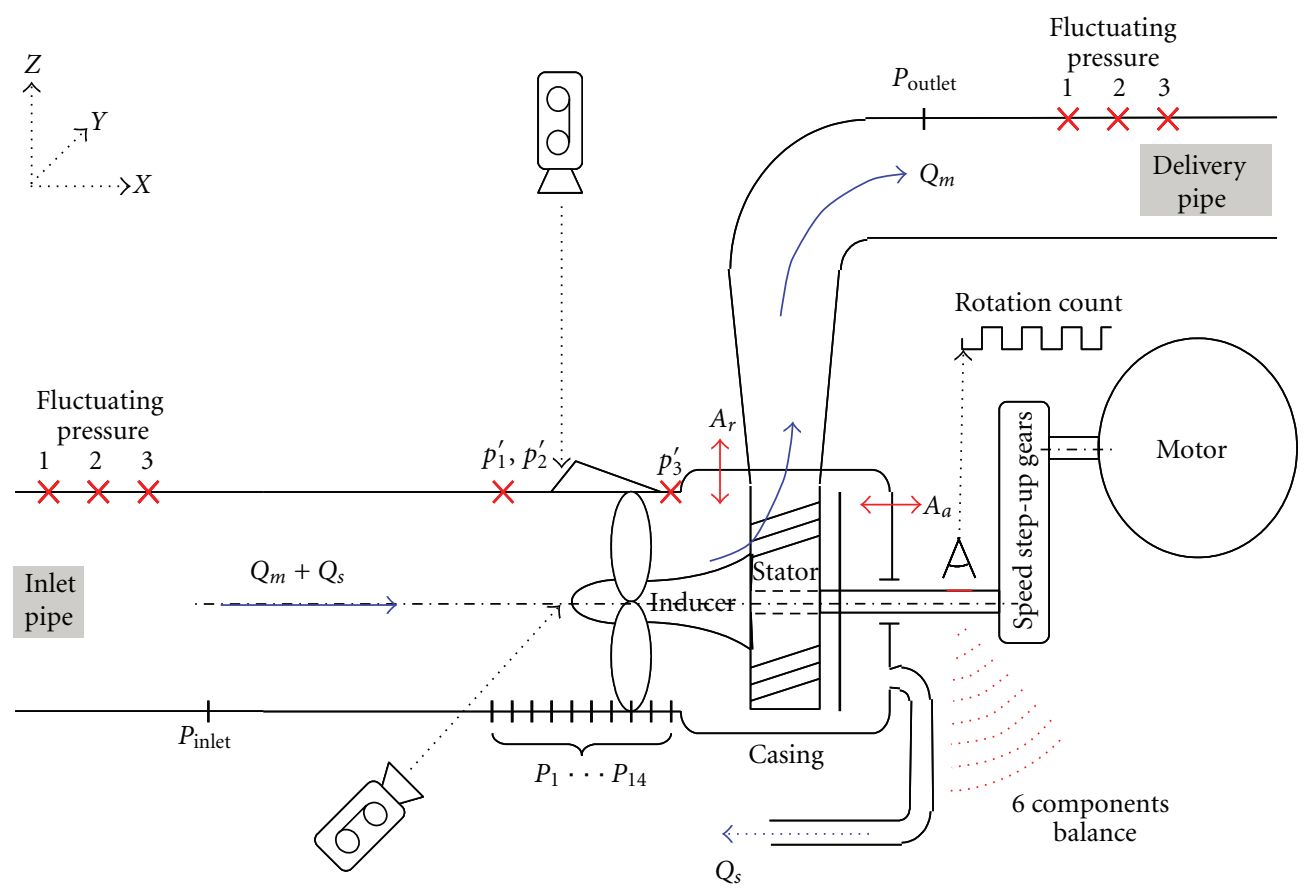

FIgURE 4: Scheme of the inducer test section including the acquisition equipment.

(i) Wavelet decomposition: this technique enables to identify on a signal the time of inception of the successive characteristic frequencies. It is based on a decomposition of the initial signal $S$ into secondary signals $S_{i}$. Each signal $S_{i}$ is obtained from a typical shape called "wavelet" and a discrete frequency corresponding to the period of the wavelet. For example, the secondary signal $S_{1}$ at frequency $f_{1}$ is composed of a succession of wavelets whose period is $1 / f_{1}$, and magnitude varies according to the consistence of the initial signal with the shape of this wavelet. The residual, that is, $S-S_{1}$ is then decomposed into a secondary signal $S_{2}$ related to frequency $f_{2}$, and a new residual that will be decomposed again and so on. The initial signal is decomposed according to several discrete frequencies, until a residual of low magnitude is obtained. If high amplitude is observed locally on a secondary signal $S_{i}$, then it means that a fluctuation at frequency close to $f_{i}$ is included in the initial signal. In the present study, decomposition into discrete wavelet (Daubechies no. 5) is applied to the pressure and force signals.

(ii) Study of the temporal signals: this method aims, through analysis of the force and pressure unsteady signals, to improve the understanding of the inception and development of the instabilities. The main objective consists in identifying transient unbalanced flow patterns, flow conditions at inception and/or vanishing of the instabilities, and phenomena related to the detected characteristic frequencies. The analysis focuses particularly on the phase difference between $F_{y}$ and $F_{z}$, or $P_{1}^{\prime}$ and $P_{2}^{\prime}$ (mounted in the same crosssection with $90^{\circ}$ difference between them). Attention is also paid to the mean values of $F_{y}$ and $F_{z}$ (the mean value of these two signals in noncavitating conditions has been set to zero).
In this analysis, low-pass filters have been applied in order to clearly distinguish some characteristic frequencies. Cut-off frequencies will be indicated systematically hereafter.

\section{Wavelet Decomposition}

The present inducer is characterized by a large number of nonsymmetrical flow patterns: so-called "synchronous" (unbalanced flow pattern rotating at inducer speed), "subsynchronous," "super-synchronous" (unbalanced flow pattern rotating slower or faster than the inducer, resp.), and alternate (unbalanced flow pattern with identical opposite sheet cavities) regimes have been identified. An unknown fluctuation at frequency $0.9 f_{\text {ref }}$ in the absolute frame has been also detected.

These flow instabilities have been identified from the waterfall plots of $F_{y}, F_{z}, P_{1}^{\prime}$, and $P_{2}^{\prime}$, as indicated in Figure 5 for $N=N_{\text {ref. }}$.

For example, super-synchronous rotating cavitation is clearly detected for $0.15<\tau^{+}<0.22$. It is identified by the low frequency that is obtained in the rotating frame from the $F_{y}$ signal, which is correlated with the peaks that can be seen from the analysis of $P_{2}^{\prime}$, at frequency $f_{\mathrm{RC}}$ higher than the one of the inducer rotation. This pattern basically consists of large-scale fluctuations of the radial force components $F_{y}$ and $F_{z}$ (Figure 6), at frequency that progressively decreases from a few tens of Hertz down to zero when $\tau^{+}$is decreased. A $90^{\circ}$ phase difference between $F_{y}$ and $F_{z}$ is observed, which suggests that these fluctuations may be associated to a rotating instability. Note that the values of the forces are not given in ordinate for confidentiality reasons: only the zero is indicated to facilitate the discussion. 

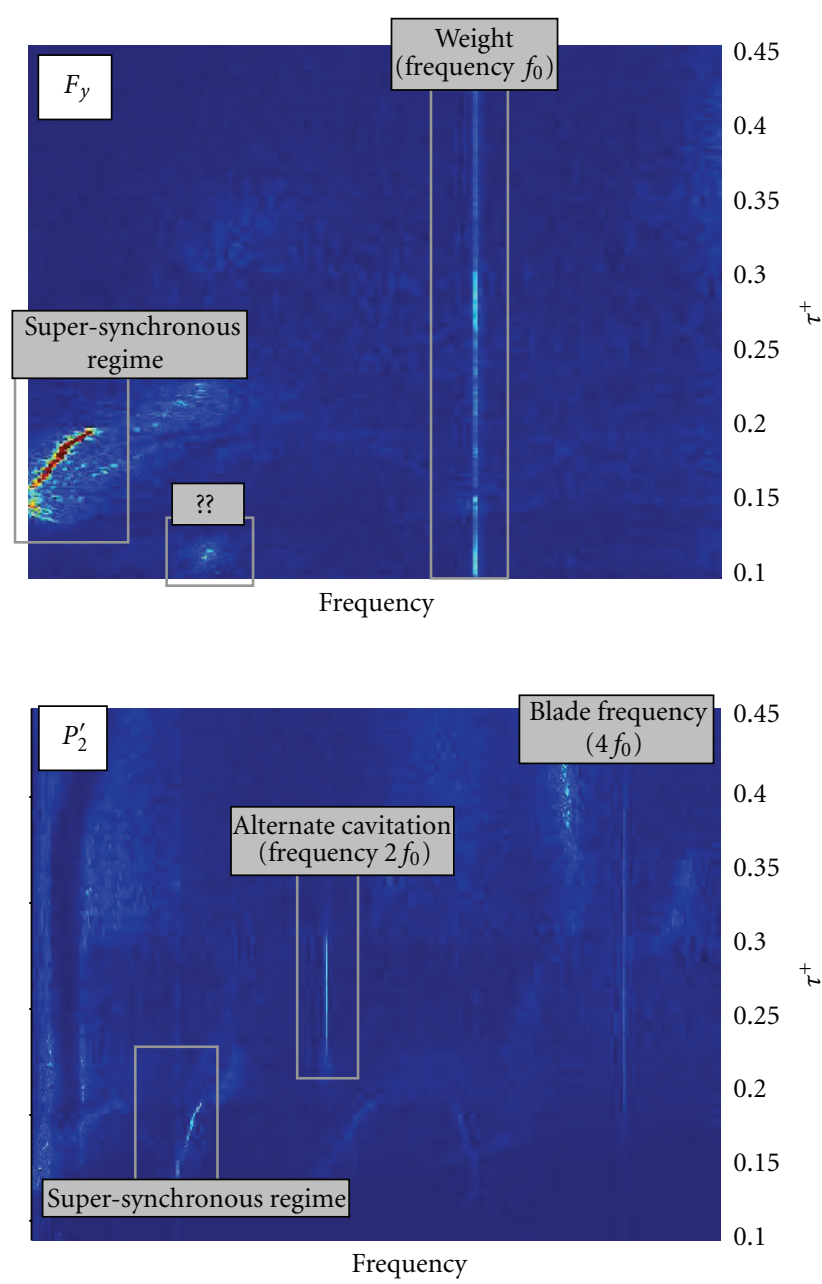

FIgURE 5: Waterfall plot for $F_{y}$ and $P_{2}^{\prime}\left(Q_{n}, N_{\text {ref }}\right)$.

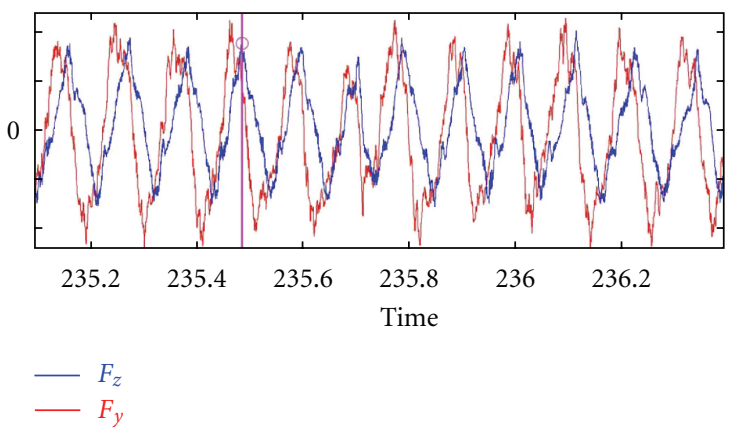

FIgURE 6: Time signals of $F_{y}$ and $F_{z}$ during the super-synchronous regime $\left(Q_{n}, N_{\text {ref }}\right.$, cut-off frequency $\left.400 \mathrm{~Hz}\right)$.

For higher cavitation number $\left(0.22<\tau^{+}<0.32\right)$, a stable nonsymmetrical flow pattern is detected. It is mainly characterized by a clear frequency $2 f_{0}$ on the spectrum analysis of $P_{1}^{\prime}$ and $P_{2}^{\prime}$ (see Figure 5), which is usually due to different sheet cavity sizes on successive blades, but identical sheet cavities on opposite blades. Since this arrangement is stable in the rotating frame, no frequency is detected in the waterfall plot of $F_{y}$ or $F_{z}$.
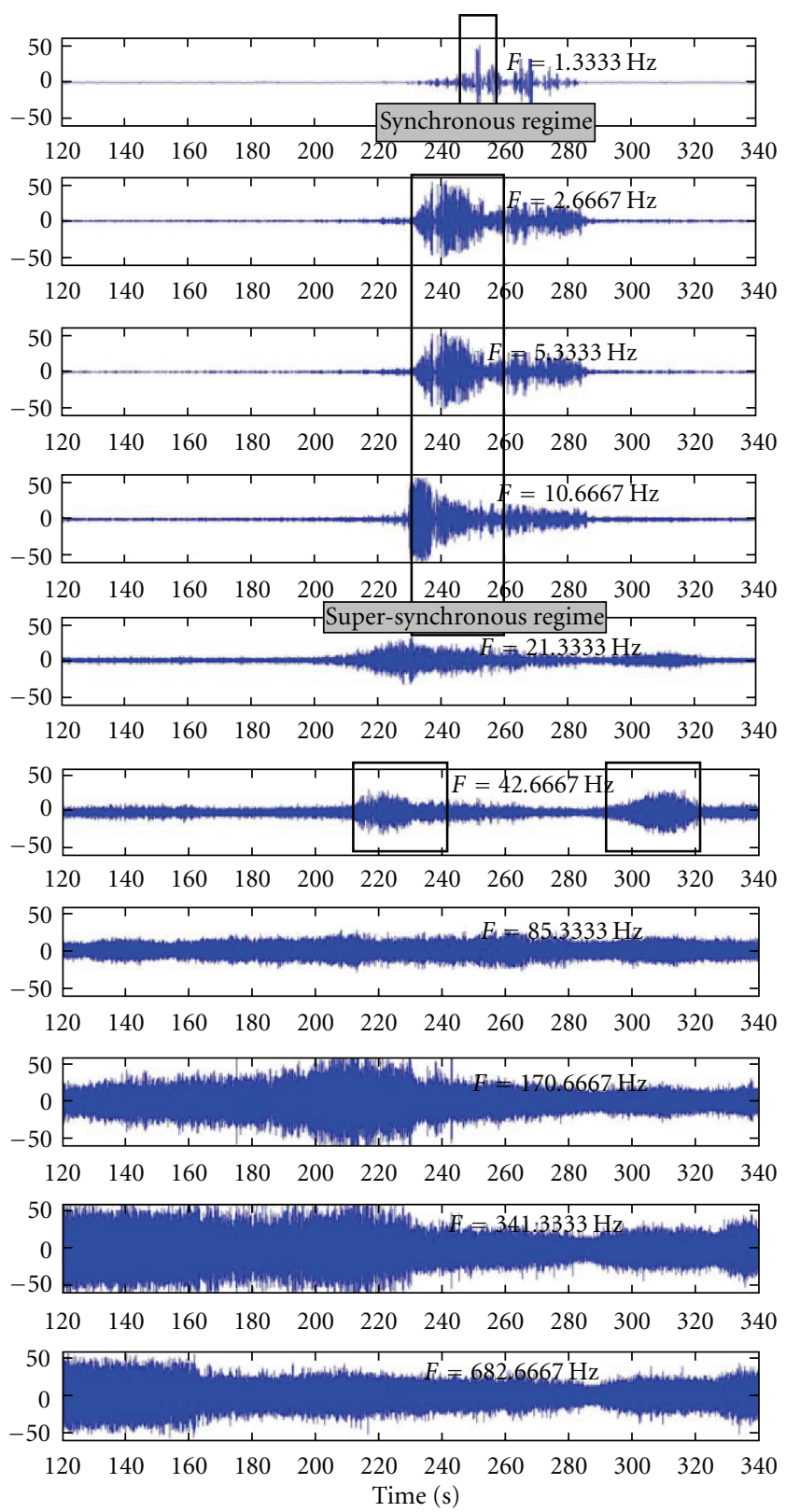

Figure 7: Wavelet decomposition of signal $F_{y}$.

In this section we focus on results at speed $1.2 N_{\text {ref, }}$ in order to have the clearest available fluctuations. Indeed, it has been shown by Coutier-Delgosha et al. (2009) that the rotation speed has some effects on the intensity of the radial loads, but does not modify the instability types.

The decomposition into discrete wavelet (Daubechies $n^{\circ} 5$ ) is applied to the $F_{y}$ signal (Figure 7). Frequency $f_{1}$ is $682 \mathrm{~Hz}$ in the present case, and $f_{10}=1.33 \mathrm{~Hz}$. The analysis is performed during the pressure decrease between $t=120 \mathrm{~s}$ and $t=340 \mathrm{~s}$, that is from the inception of the first instability regime (alternate cavitation) to the performance breakdown. For high frequencies, high amplitude is nearly systematically obtained, whereas below $100 \mathrm{~Hz}$, several points can be noticed. 


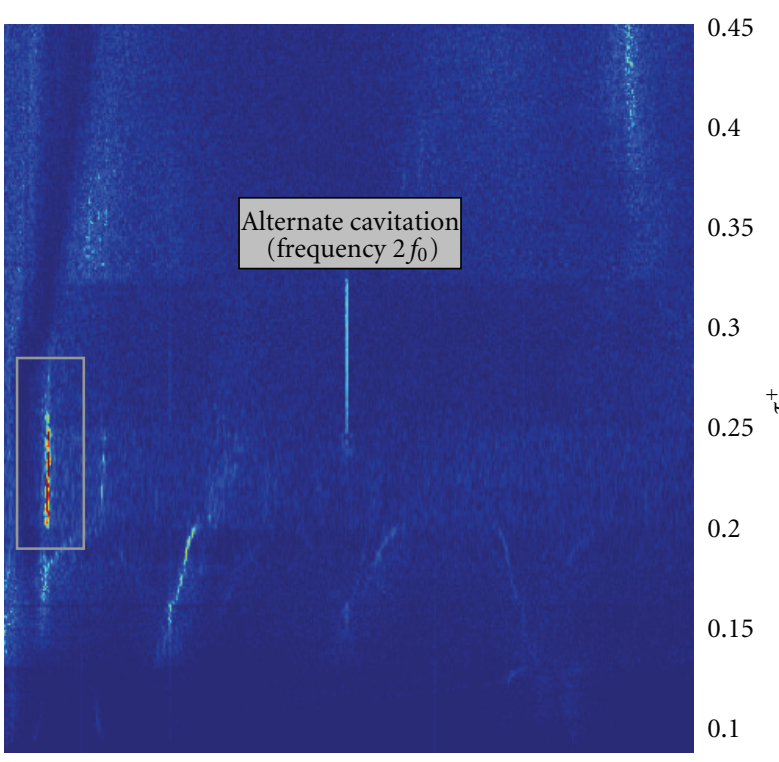

Frequency

(a)

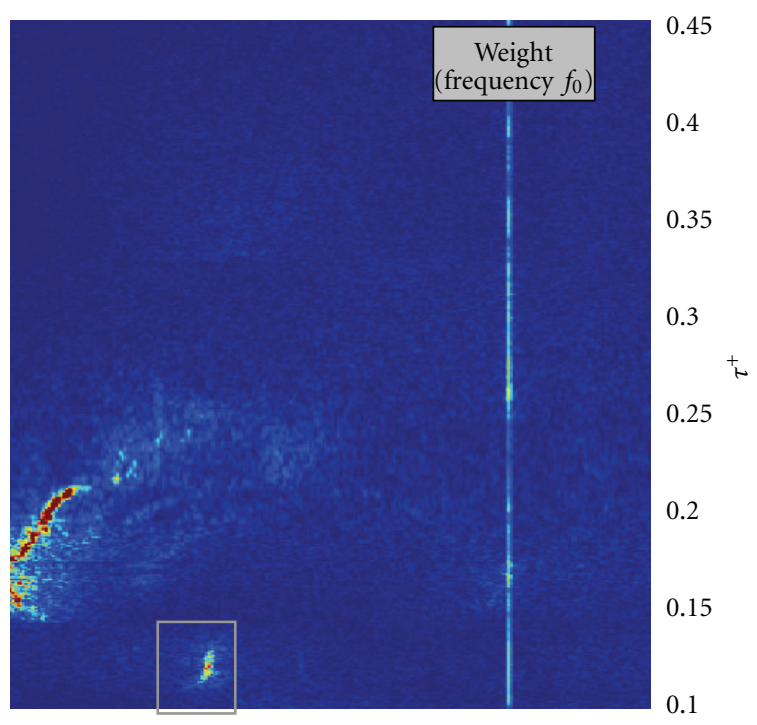

Frequency

(b)

Figure 8: Waterfall plot of (a) $P_{2}^{\prime}$ and (b) $F_{y}\left(Q_{n}, 1.2 N_{\text {ref }}\right)$.

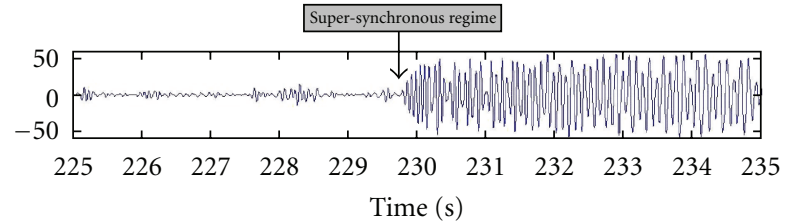

Figure 9: Wavelet decomposition for $F_{y}$. Zoom on the time interval $225 \mathrm{~s}<t<235 \mathrm{~s}$ for $f=10.66 \mathrm{~Hz}\left(N_{\text {ref }}, Q_{n}\right)$.

(i) Close to $40 \mathrm{~Hz}$, two sequences are characterized by a significant increase of the intensity: between $t=210 \mathrm{~s}$ and $230 \mathrm{~s}\left(0.19<\tau^{+}<0.23\right)$, and between $t=300 \mathrm{~s}$ and $320 \mathrm{~s}\left(0.075<\tau^{+}<0.09\right)$.

(ii) The inception of the super-synchronous instability can be observed clearly in the decomposition at frequency $f=10.66 \mathrm{~Hz}$, and also in the adjacent decompositions, with a lower intensity. It is observed that this characteristic frequency progressively decreases, until a short synchronous regime (which can be detected here at the lowest frequency) is obtained, for $253 \mathrm{~s}<t<255 \mathrm{~s}$.

The same process is applied to the unsteady pressure $P_{2}^{\prime}$. The main resulting information is a very clear unsteadiness characterized by a frequency close to $20 / 40 \mathrm{~Hz}$ for $210 \mathrm{~s}<$ $t<230 \mathrm{~s}$. Such frequency was already observed during the same time on the $F_{y}$ signal, but not so clearly. Figure 7 shows the waterfall plot for $P_{2}^{\prime}$ at rotation speed $1.2 N_{\text {ref. A }}$ high-intensity signal at frequency close to $30 \mathrm{~Hz}$ is obtained, between the regime of alternate cavitation and the inception of the super-synchronous instability, that is, for $0.19<$ $\tau^{+}<0.23$. This corresponds to $210 \mathrm{~s}<t<230 \mathrm{~s}$. It can be noticed that this frequency was much weaker at rotation speed $N_{\text {ref }}$ (see Figure 5). Similarly, the second instability regime detected on the $F_{y}$ wavelet decomposition (for $300 \mathrm{~s}$ $<t<320 \mathrm{~s}$ ) clearly appears on the waterfall plot at $1.2 \mathrm{~N}_{\text {ref, }}$ while it can hardly be detected at speed $N_{\text {ref }}$ (cf. Figure 5).

It can be concluded that the wavelet decomposition does not enable any better understanding of the mechanisms related to the different instabilities. Conversely, it can be used to detect precisely their inception, as can be seen for example in Figure 9 in the case of the super-synchronous regime.

\section{Analysis of the Temporal Signals}

Several types of instabilities are encountered during the process of pressure decrease at $1.2 N_{\text {ref }}$ and $Q_{n}$. In the present paper, attention is focused on the regime of alternate cavitation.

This regime is obtained for $130 \mathrm{~s}<t<210 \mathrm{~s}$, that is, for $0.23<\tau^{+}<0.35$. It is characterized by sheet cavities of different sizes on two consecutive blades but identical on opposite ones. This nonsymmetrical arrangement results in a clear frequency $2 f_{0}$ on the spectrum analysis of $P_{1}^{\prime}$ and $P_{2}^{\prime}$ (see Figure 8). Conversely, no evidence of this unbalanced flow pattern can be found in the waterfall plot of $F_{y}$ or $F_{z}$, since it is stable in the inducer rotating frame.

Supplementary information can be obtained in the present case from the analysis of the temporal signals: if a low-pass filter with cut-off frequency $100 \mathrm{~Hz}$ is applied to the signals of $F_{y}$ and $F_{z}$, in order to get rid of the high-frequency fluctuations, it becomes clear that mean values of $F_{y}$ and $F_{z}$ are significantly different during all the regime (Figure 10). Note that the values of the forces are not given in ordinate for 


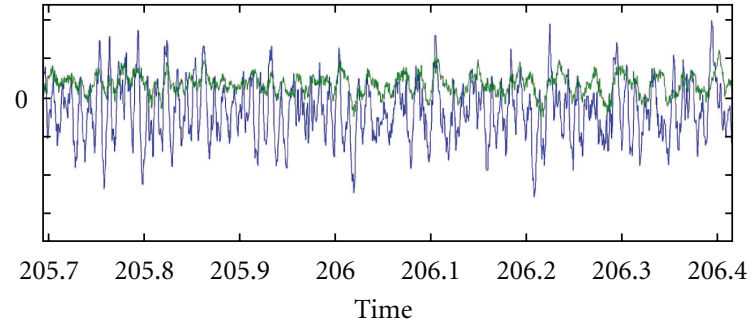

Figure 10: Temporal signals of $F_{y}$ and $F_{z}$ with low pass filter (cutoff frequency $100 \mathrm{~Hz}), Q_{n}, 1.2 \mathrm{~N}_{\text {ref. }}$.

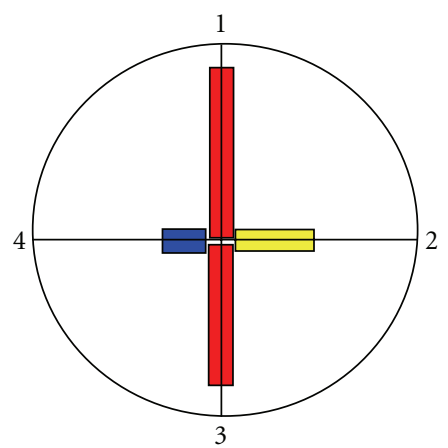

Figure 11: Plausible configuration for $0.23<\tau^{+}<0.35$. Rectangles represent schematically the respective lengths of the sheet cavities on the four blades $\left(1.2 N_{\text {ref }}, Q_{n}\right)$.

confidentiality reasons: only the zero is indicated to facilitate the discussion.

Although the values of the forces are not given in ordinate for confidentiality reasons, it is clear that $F_{y}$ fluctuates around a negative average value $-F_{\text {mean }}$, while $F_{z}$ is characterized by a positive mean value close to $F_{\text {mean }}$. It implies that the present nonsymmetrical flow pattern causes in addition a constant mean radial force, which does not rotate in the inducer rotating frame. It means that cavitation on opposite blades may not be fully identical. However, the $2 f_{0}$ frequency on the pressure waterfall plots shows that two identical cavitation patterns separated by $180^{\circ}$ are also present inside the inducer. The term "cavitation pattern" is voluntary used, since it may concern too sheet cavitation on the blade suction side as tip cavitation close to the inducer shroud.

The most plausible cavitation pattern is thus composed of two identical cavitation areas on two opposite blades ( 1 and 3 ), while the two other ones (2 and 4) are both of different size. In this configuration, a constant radial load is obtained in the inducer rotating frame because of the dissymmetry between blades 2 and 4. This load, represented schematically in Figure 11, may result (excepted in a particular orientation of axes $y$ and $z$ ) in different mean values of the components $F_{y}$ and $F_{z}$.

Another feature of the alternate cavitation regime can be remarked on the extended waterfall plots of $F_{y}$ or $F_{z}$ (see Figure 12). Frequency exactly equal to $3 f_{0}$ is obtained for $0.23<\tau^{+}<0.35$.

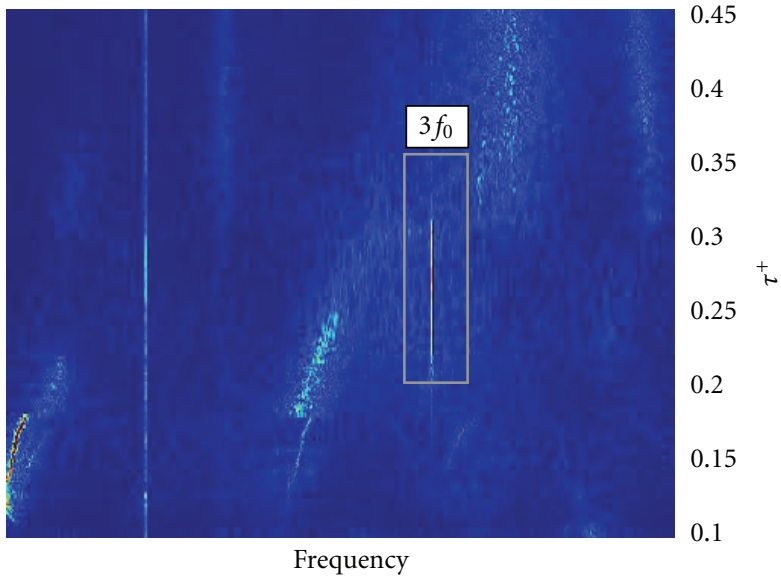

FIgURE 12: Extended waterfall plot of $F_{y}\left(Q_{n}, N_{\text {ref }}\right)$.

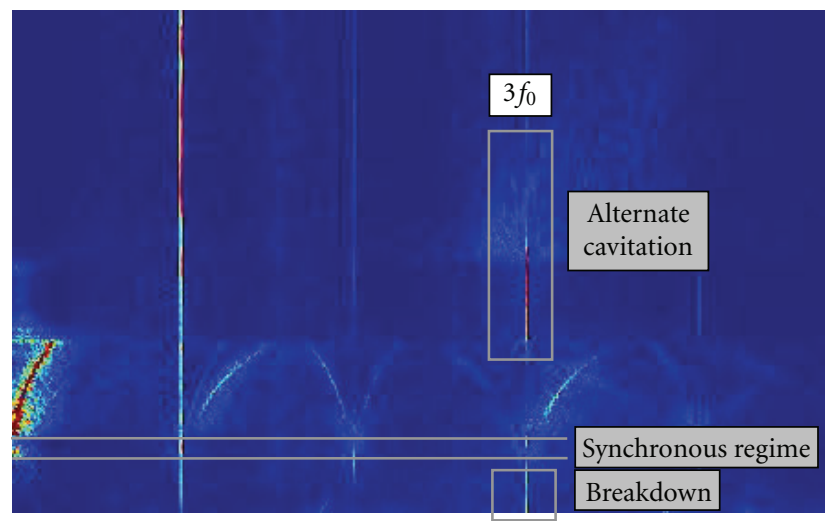

Frequency

Figure 13: Extended waterfall plot of $F_{y}\left(1.18 Q_{n}, N_{\text {ref }}\right)$.

The following points can be checked from the analysis of the waterfall plots obtained at various rotation speeds and mass flow rates.

(i) It is a $3 f_{0}$ frequency, which changes with the inducer rotation speed.

(ii) It can be detected mainly above the nominal flow rate, that is, within the range of mass flow rate that leads to most of the regimes of radial loads. It is particularly strong at high mass flow rate (see Figure 13).

(iii) It is not specific to the alternate cavitation flow pattern: all regimes of developed and stable (i.e. nonrotating) cavitation result in the occurrence of the $3 f_{0}$ frequency. It is observed for example in the case presented in Figure $13\left(N_{\text {ref }}, 1.18 Q_{n}\right)$ that this frequency is obtained during three successive periods: (1) alternate cavitation, (2) short synchronous regime that occurs just after the super-synchronous one, and (3) regime of highly developed cavitation during the final performance breakdown. 


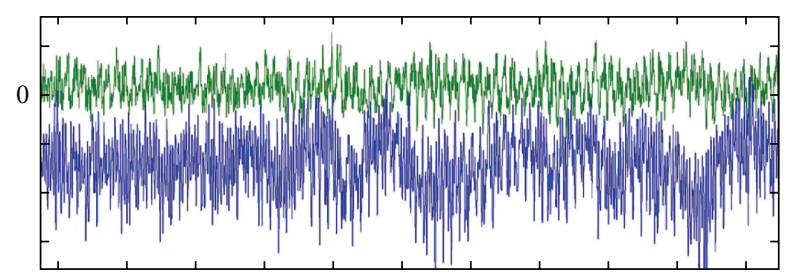

$265.1265 .2265 .3265 .4265 .5265 .6265 .7265 .8265 .9266 \quad 266.1$

Time

Figure 14: Temporal signals of $F_{y}$ (in blue) and $F_{z}$ (in green) $\left(N_{\text {ref }}\right.$, $1.18 Q_{n}$ ).

(iv) The $3 f_{0}$ frequency can be detected even in noncavitating conditions, but strongly attenuated.

From these observations, no obvious explanation can be found regarding the occurrence of the $3 f_{0}$ frequency. Conversely, some points enable to improve the understanding of this phenomenon. For example, no peak at frequency $3 f_{0}$ is observed on the waterfall plots of the radial load intensity $\mathrm{Fr}$, whatever the flow configuration is. It means that the radial load does not fluctuate; only its components do. Besides, the $3 f_{0}$ frequency is clearly detected on the waterfall plots of the bending moments $M_{y}$ and $M_{z}$, which suggests that this instability is related to a shaft bend stress. On the other hand, the frequency peak is very small on the waterfall plots of the axial load $F_{x}$, which eliminates any direct connection with an axial effort on the shaft.

Three different hypotheses can be postulated.

(i) A possible explanation is a rotating perturbation that would rotate at frequency $4 f_{0}$ in the reference steady frame, which would generate a frequency $3 f_{0}$ in the inducer rotating frame. Such phenomenon has been observed in experiments conducted in the Osaka University with a three-blade inducer, resulting in a $4 f_{0}$ frequency in the inducer frame [10]. It is called high-order rotating cavitation by Tsujimoto and colleagues. Although the present detected frequency is not the same, this example shows that the hypothesis of a perturbation with high rotation speed is plausible. However, it is not the most probable one, because of the exact value $3 f_{0}$ that is obtained.

(ii) It can be the result of a rotor/stator interaction at the inducer outlet. A method proposed by Brennen [18] enables to obtain the characteristic frequencies related to such interaction. In the present case, the stator in the discharge pipe is equipped with 13 blades, the inducer has 4 blades, so frequencies $f_{0}, 2 f_{0}, 3 f_{0}$, and $4 f_{0}$ should appear on the plots with similar intensities. So, frequency $3 f_{0}$ should not be predominant. Still it must be noticed that a frequency range $250 \mathrm{~Hz}-300 \mathrm{~Hz}$ has been previously identified as resonant frequencies of the installation. The $3 f_{0}$ peak may thus be of higher intensity than the other ones if it belongs to this range. However this hypothesis is also rejected, because the $3 f_{0}$ peak is of high intensity for all rotation speeds, from $0.6 N_{\text {ref }}$ up

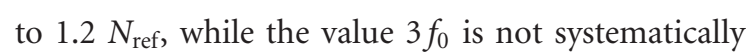
included into the range of resonant frequencies.

(iii) It can be related to a fluctuation of the radial load angle during the inducer rotation. More precisely, it can be anticipated that several fluctuations occurring during each inducer revolution may result in the measurement of the frequency $3 f_{0}$. To investigate this point, a more in-depth analysis of the temporal signals of $F_{y}$ and $F_{z}$ has been conducted during the alternate cavitation regime for flow conditions $N_{\text {ref }}$ and $1.18 Q_{n}$, where frequency $3 f_{0}$ is remarkably clear on the waterfall plots. Figure 14 presents the general aspect of the two signals ( $F_{y}$ in grey $F_{z}$ in black), which confirms the difference of mean level that was mentioned previously.

A zoom on a shorter time period is performed in Figure $15(\mathrm{a})$, to obtain only 25 rotations of the inducer. Oscillations at frequency $f_{0}$ can be clearly observed on both signals. Two secondary oscillations of lower amplitude are also detected on the $F_{y}$ signal, which may explain the presence of frequency $3 f_{0}$ in the spectrum. In the case of $F_{z}$, these secondary oscillations do not appear so clearly. A second zoom is performed to focus on six rotations of the inducer only (see Figure 15(b)). Regular oscillations at frequencies $f_{0}$ and $3 f_{0}$ are observed: the two secondary peaks are regularly positioned between the main oscillations. On this figure, secondary peaks can be also seen in the signal of component $F_{z}$ (Figure 15(c)). It can be also noticed that the phase difference between $F_{y}$ and $F_{z}$ varies.

(i) The phase difference at low frequency $f_{0}$, which corresponds to the gap between the vertical full lines in Figures 15(b) and 15(c), is small. It equals $90^{\circ}$, since it is related to the inducer weight.

(ii) The phase difference between the secondary peaks is not the same. It can be estimated that it is close to $180^{\circ}$. It means that the phenomenon that generates the secondary oscillations results in fluctuations of $F_{y}$ and $F_{z}$ characterized by phase opposition. It must be mentioned that the analysis of various periods has enabled to draw this conclusion, while interpretation of Figures 15(b) and 15(c) is not so clear. On the basis of these observations, no definitive interpretation can be found for frequency $3 f_{0}$.

However, a possible explanation, which could be validated only from high-speed visualizations, is given hereafter. It is based on a probable oscillation of the two sheet cavities of different sizes no. 2 and no. 4 denoted in Figure 11. These two areas may fluctuate around a mean size, and even reverse (i) because of the vertical pressure gradient due to the gravity within the inducer. The Froude number based on the inducer diameter and the axial velocity is about 0.2 , which confirms that the gravity may have some influence on the flow during its passage in the machinery; (ii) or by effect of the twophase structures which may be different at the top and at the 


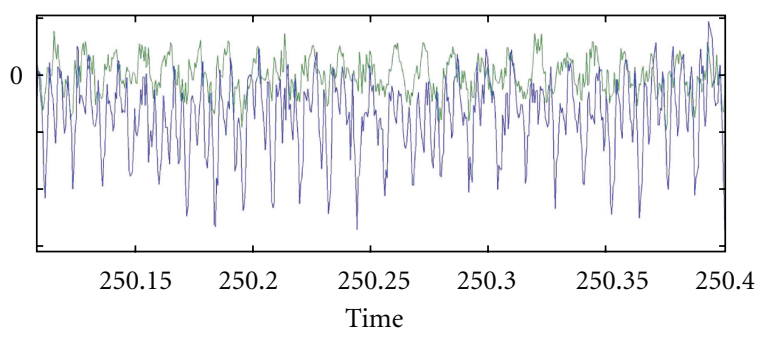

(a)

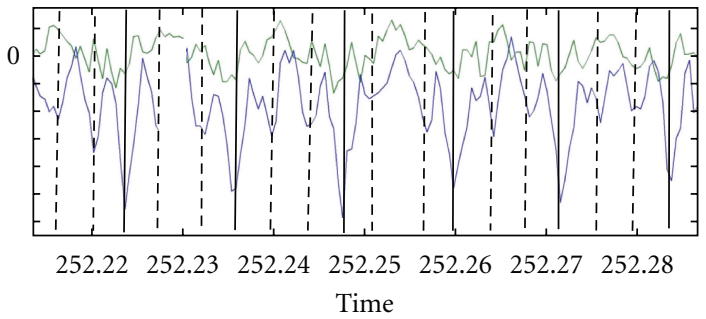

(b)

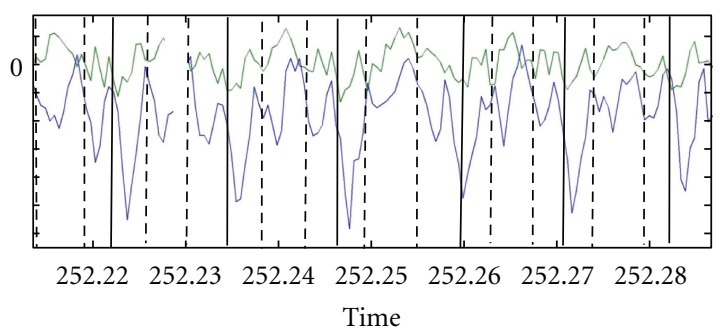

(c)

Figure 15: Zoom on the signals of $F_{y}$ (in blue) and $F_{z}$ (in green), $1.18 Q_{n}, N_{\text {ref }}$, and low pass filter with cut-off frequency $400 \mathrm{~Hz}$.

bottom of the inducer, which would be also an indirect effect of the gravity.

In this case, the oscillation of components $F_{y}$ and $F_{z}$ at frequency $f_{0}$, due to the inducer weight, may be coupled to this inversion of sheet cavities 2 and 4, which would occur twice during each rotation of the inducer, leading to a more complex signal. A scheme of the evolutions of $F_{y}$ and $F_{z}$ during a single rotation of the inducer is proposed in Figure 17. It is supposed here that the radial load related to the nonsymmetry between blades 2 and 4 is oriented according to the scheme given in Figure 16.

This particular orientation of $F_{y}$ and $F_{z}$ enables to explain the $180^{\circ}$ phase difference between the two components in the secondary oscillations. Indeed, in such configuration, the inversion of the sheet cavities 2 and 4, which makes an inversion of the associated radial load, results in opposite variations of $F_{y}$ and $F_{z}$. It must be noticed that the blade orientations drawn in Figure 17 represent schematically the direction of the radial load generated by nonidentical sheet cavities on opposite blades. It can be anticipated that these directions are close to the lines that would join the leading edge of the opposite blades.

It is observed in Figure 15 that the oscillations of $F_{y}$ are of larger amplitude than those of $F_{z}$, which suggests that the angle between each component and the blades is not $45^{\circ}$. As indicated in Figure 16, the direction of $F_{y}$ may be close to the direction of the radial load related to the nonsymmetrical flow pattern on blades 2 and 4. The expected evolutions of $F_{y}$ and $F_{z}$ during a single inducer rotation are drawn in Figure 17 on the basis of this hypothesis.

The eight schemes at the top of Figure 16 show the eight successive positions of sheet cavities on blades 2 and 4 , during a revolution of the machinery, by step of $45^{\circ}$. It can be observed that two inversions of the small and big sheet

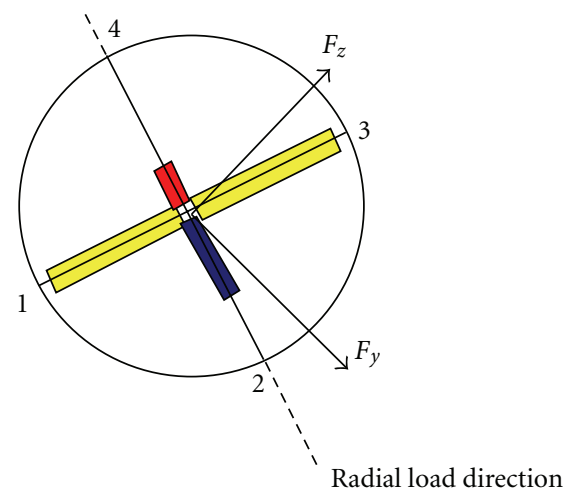

Figure 16: Expected orientation of $F_{y}$ and $F_{z}$, and the blades (1.18 $\left.Q_{n}, N_{\text {ref }}\right)$.

cavities occur during one period, each time they cross the horizontal direction. Between two inversions, the difference of size between sheet cavities 2 and 4 first increases reaching a maximal value, and then decreases. It is maximal when the radial load is vertical, since in such configuration the pressure gradient, which controls the pulsation of the cavities according to our assumption, has the strongest effect on the sheet cavities located on blades 2 and 4 .

On the central part of Figure 17, the curves in full line correspond to the evolutions of $F_{y}$ and $F_{z}$ without the radial load generated by the unbalanced flow pattern on blades 2 and 4: a sinusoidal evolution; due to the respective orientations of the gravity, $F_{y}$, and $F_{z}$, with a $\pi / 2$ phase difference between the load components, is obtained in that case. This behaviour is modified by the fluctuations of the sheet cavities on blades 2 and 4, leading to the curves drawn in doted lines. 

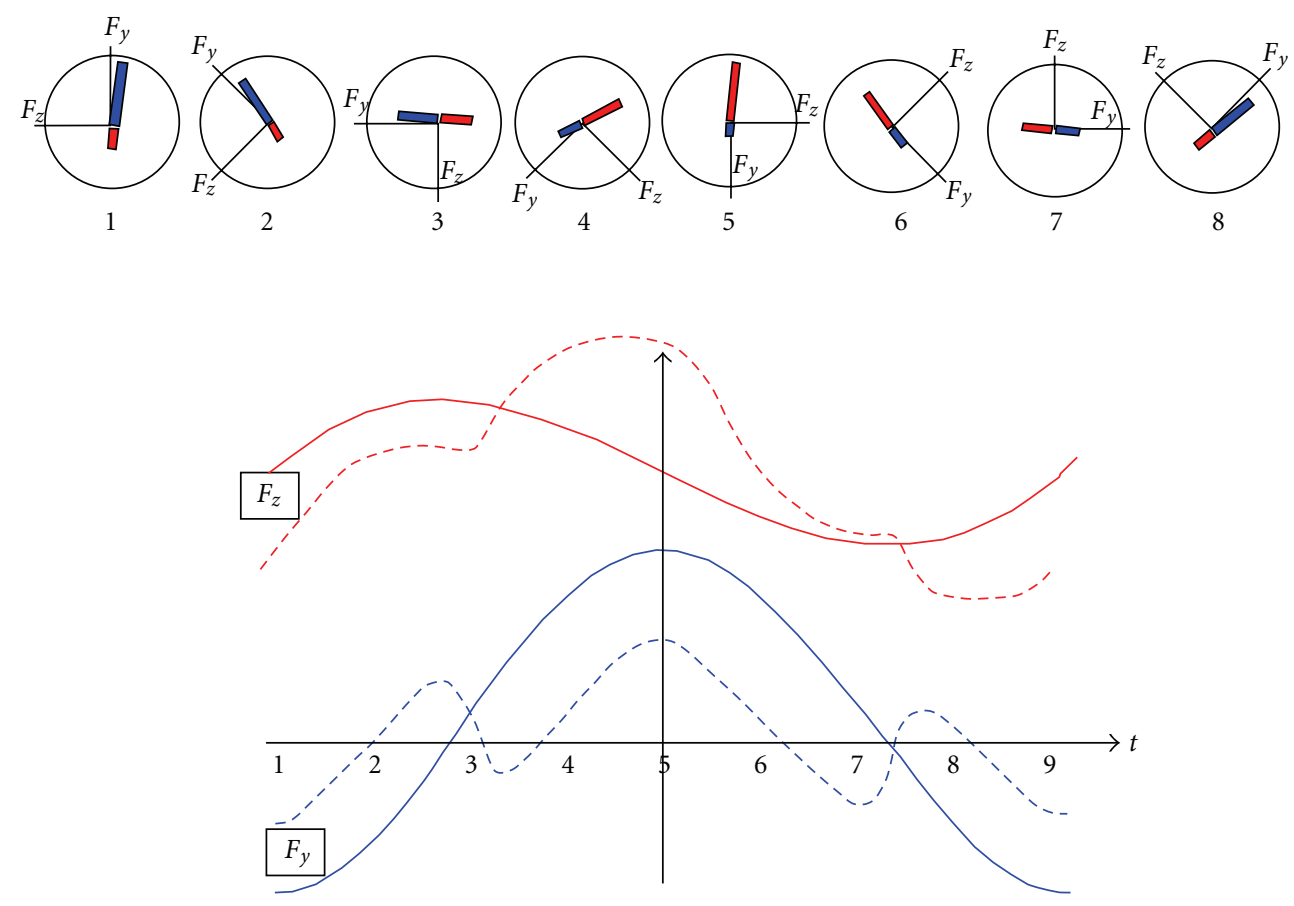

FIGURE 17: Scheme of the qualitative evolutions of $F_{y}$ (blue curve) and $F_{z}$ (red curve) during a single rotation of the inducer.

The evolution of $F_{y}$ is composed of the following steps.

(i) It can be anticipated that the radial load will systematically be oriented from the small cavity towards the big one (i.e., from high-pressure areas towards low pressures). So, configuration 1 in Figure 17 is characterized by a component $F_{y}$ related to the flow dissymmetry that is positive, and even almost maximal, since blades 2 and 4 are close to the vertical direction. Between steps 1 and 3, the radial load reaches its maximal value, and then it decreases, so the curves in full and dotted lines become closer. It must be reminded that all charts here focus on a qualitative behaviour, while the real evolutions of $F_{y}$ and $F_{z}$ may depend strongly on the relative values of the radial load related to the inducer weight and the one related to nonsymmetrical cavitation on blades 2 and 4 .

(ii) Just after configuration 3, blades 2 and 4 cross the horizontal direction, so an inversion of the sheet cavity lengths is obtained. Consequently, the radial load due to the dissymmetry, and thus the sign of the $F_{y}$ component related to this load, is also inverted. It results in a sudden decrease of $F_{y}$, under the curve in full line. Then, $F_{y}$ reincreases progressively until step 5 is reached, since its direction becomes closer to the one of the gravity. In the same time, the radial load due to the dissymmetry also increases, but it is negative, so it slightly counterbalances the effect of the inducer weight.

(iii) Steps 6 to 8 lead to the same variations of $F_{y}$, but in the opposite direction: between steps 5 and 6 , components related to the inducer weight and to the dissymmetry are both decreased. When the leading edges of blades 2 and 4 cross the horizontal direction (just after configuration 7), sheet cavities invert again, so the radial load that they generate also does, and $F_{y}$ increases over the full line curve. Then, the effect of the weight decreases, leading to the decrease of $F_{y}$.

The curve for $F_{z}$ is obtained by a similar process: from steps 1 to 3 , the radial load due to sheet cavities on blades 2 and 4 is negative and decreases. After configuration 3, it inverts, so it becomes positive and increases more and more when the sheet cavities get closer to the vertical direction. After configuration 5, the radial load decreases again, and $F_{z}$ also does and comes closer to the curve in full line. Just after step 7 , sheet cavities invert again, and the $F_{z}$ component related to the dissymmetry becomes negative and decreases down to its value at step 1 .

This scenario generates for both components $F_{y}$ and $F_{z}$ three successive fluctuations during each rotation of the inducer, which is consistent with frequency $3 f_{0}$ that is observed on the waterfall plots. In the configuration presented in Figure 17, the three pulsations of $F_{y}$ occur at equal distance to each other, which explains the intense peak at frequency $3 f_{0}$. Oscillations of component $F_{z}$ are not so regular during one rotation, which is consistent with observations from Figure 15, and which also explains that the spectral line at $3 f_{0}$ is weaker for $F_{z}$ than for $F_{y}$.

\section{Conclusion}

The instabilities detected in a four-blade inducer have been discussed in the present paper by wavelet decomposition and 
analysis of the temporal signals of the two components $F_{y}$ and $F_{z}$ of the radial load on the shaft. It has been found that wavelet decomposition does not improve the understanding of the flow instabilities, while it enables a precise detection of inception and stop of each unbalanced flow pattern. An unexpected spectral line at $3 f_{0}$, which occurs during alternate cavitation, has been discussed by direct analysis of the temporal signals of $F_{y}$ and $F_{z}$. A possible scenario, based on the assumption that the gravity may have-directly or indirectly-some effect on the sheet cavities on the blades, is proposed. Only high-speed video would enable to confirm this hypothesis.

\section{Nomenclature}

$f: \quad$ Frequency $(\mathrm{Hz})$

$f_{0}$ : Frequency of the inducer rotation $(\mathrm{Hz})$

$F_{x}$ : Axial component of the force on the shaft $(\mathrm{N})$

$F_{y}, F_{z}$ : Radial components of the force on the shaft $(\mathrm{N})$

$N$ : $\quad$ Rotation speed $\left(\mathrm{s}^{-1}\right)$

$N_{\text {ref: }}$ Reference rotation speed $\left(\mathrm{s}^{-1}\right)$

$\Delta P: \quad$ Pressure elevation $P_{d}-P_{i}(\mathrm{~Pa})$

$P_{i}$ : $\quad$ Absolute pressure in the inlet pipe $(\mathrm{Pa})$

$P_{d}: \quad$ Absolute pressure in the delivery pipe $(\mathrm{Pa})$

$P t_{i}: \quad$ Total absolute pressure in the inlet pipe $(\mathrm{Pa})$

$P t_{d}$ : Total absolute pressure in the delivery pipe $(\mathrm{Pa})$

$P_{\text {vap: }}$ Vapor pressure $(\mathrm{Pa})$

$P_{1}^{\prime}, P_{2}^{\prime}$ : Fluctuating pressures at the inducer inlet $(\mathrm{Pa})$

$P_{3}^{\prime}$ : $\quad$ Fluctuating pressure at the inducer outlet $(\mathrm{Pa})$

$M_{x}$ : Torque on the inducer shaft (Nm)

$M_{y, z}$ : Bending moments on the shaft $(\mathrm{Nm})$

$Q: \quad$ Volume flow rate $\left(\mathrm{m}^{3} / \mathrm{s}\right)$

$Q_{n}: \quad$ Nominal flow rate $\left(\mathrm{m}^{3} / \mathrm{s}\right)$

$Q_{m}$ : Main flow rate (in the delivery pipe) $\left(\mathrm{m}^{3} / \mathrm{s}\right)$

$Q_{s}: \quad$ Secondary flow rate (for axial equilibrium) $\left(\mathrm{m}^{3} / \mathrm{s}\right)$

$r: \quad$ Inducer tip radius $(\mathrm{m})$

$t: \quad$ Time (s)

$v$ : $\quad$ Outlet ratio hub radius/tip radius $(-)$

$\Psi: \quad$ Head coefficient $\left(P t_{d}-P t_{i}\right) /\left(\rho \omega^{2} r^{2}\right)(-)$

$\chi: \quad$ Torque coefficient $M_{x} /\left(\rho \omega^{2} r^{5}\right)(-)$

$\phi: \quad$ Flow rate coefficient $\mathrm{Q} /\left(\pi \omega r^{3}\left(1-v^{2}\right)\right)(-)$

$\tau: \quad$ Cavitation number $\left(P t_{i}-P_{\text {vap }}\right) /\left(\rho \omega^{2} r^{2}\right)(-)$.

\section{Acknowledgments}

The present study was performed in the frame of contractual activity with SNECMA Moteurs and the CNES (French Space Agency). The technical staff of the LML laboratory was much involved in the development and the operation of the test facility. The authors wish to thank especially J. Choquet and P. Olivier for their collaboration.

\section{References}

[1] K. Kamijo, T. Shimura, and M. Watanabe, "An experimental investigation of cavitating inducer Instability," ASME Paper 77-WA/FW-14, 1977.

[2] B. Goirand, A. Mertz, F. Joussellin, and C. Rebattet, "Experimental investigation of radial loads induced by partial cavitation with liquid hydrogen inducer," in Proceedings of the 3rd International Conference on Cavitation (ImechE '92), vol. C453/056, pp. 263-269, Cambridge, UK, 1992.

[3] J. de Bernardi, F. Rossellini, and A. Von Kaenel, "Experimental analysis of instabilities related to cavitation in turbopump inducer," in Proceedings of the 1st International Symposium on Pump Noise and Vibrations, pp. 91-99, Paris, France, 1993.

[4] R. A. Furness and S. P. Hutton, "Experimental and theoretical studies of two-dimensional fixed-type cavities," vol. 97, pp. 515-522, 1975.

[5] Q. Le, J. P. Franc, and J. M. Michel, "Partial cavities: global behavior and mean pressure distribution," ASME Transactions Journal of Fluids Engineering, vol. 115, no. 2, pp. 243-248, 1993.

[6] J. B. Leroux, O. Coutier-Delgosha, and J. A. Astolfi, "A joint experimental and numerical study of mechanisms associated to instability of partial cavitation on two-dimensional hydrofoil," Physics of Fluids, vol. 17, no. 5, article 052101, pp. 1-20, 2005.

[7] O. Coutier-Delgosha, B. Stutz, A. Vabre, and S. Legoupil, "Analysis of cavitating flow structure by experimental and numerical investigations," Journal of Fluid Mechanics, vol. 578, pp. 171-222, 2007.

[8] Y. Tsujimoto, "Cavitation instabilities in inducers," Tech. Rep. AVT-143 RTO AVT/VKI Lecture Series, von Karman Institute, Rhode-Saint-Genèse, Belgium, 2006.

[9] F. Joussellin, J. De Bernardi, B. Goirand, and Y. Delannoy, "Analyse par films ultrarapides de poches de cavitation sur l'Inducteur de la Turbopompe à Hydrogène d'un Moteur Fusée," in 5ème colloque de visualisation et de traitement d'images en Mécanique des Fluides, Poitiers, France, 1992.

[10] A. Fujii, S. Azuma, Y. Yoshida, Y. Tsujimoto, H. Horiguchi, and S. Watanabe, "Higher order rotating cavitation in an inducer," International Journal of Rotating Machinery, vol. 10, no. 4, pp. 241-251, 2004.

[11] O. Coutier-Delgosha, Y. Courtot, F. Joussellin, and J. L. Reboud, "Numerical simulation of the unsteady cavitation behavior of an inducer blade cascade," AIAA Journal, vol. 42, no. 3, pp. 560-569, 2004.

[12] Y. Yoshida, Y. Sasao, K. Okita, S. Hasegawa, M. Shimagaki, and T. Ikohagi, "Influence of thermodynamic effect on synchronous rotating cavitation," ASME Transactions Journal of Fluids Engineering, vol. 129, no. 7, pp. 871-876, 2007.

[13] F. Bakir, S. Kouidri, R. Noguera, and R. Rey, "Experimental analysis of an axial inducer influence of the shape of the blade leading edge on the performances in cavitating regime," ASME Transactions Journal of Fluids Engineering, vol. 125, no. 2, pp. 293-301, 2003.

[14] Y. Yoshida, Y. Tsujimoto, D. Kataoka, H. Horiguchi, and F. Wahl, "Effects of alternate leading edge cutback on unsteady cavitation in 4-bladed inducers," ASME Transactions Journal of Fluids Engineering, vol. 123, no. 4, pp. 762-770, 2001.

[15] Y. D. Choi, J. Kurokawa, and H. Imamura, "Suppression of cavitation in inducers by J-Grooves," ASME Transactions Journal of Fluids Engineering, vol. 129, no. 1, pp. 15-22, 2007.

[16] H. Horiguchi, T. Takashina, and Y. Tsujimoto, "Theoretical analysis of cavitation in inducers with unequally spaced blades," JSME International Journal, Series B, vol. 49, no. 2, pp. 473-481, 2006.

[17] O. Coutier-Delgosha, G. Caignaert, G. Bois, and J. B. Leroux, "Influence of the blade number on inducer cavitating behavior," Journal of Fluids Engineering. In press.

[18] C. E. Brennen, "Pump vibration, concepts," in Hydrodynamics of Pumps, chapter 8, NREC and Oxford University Press, 1994. 

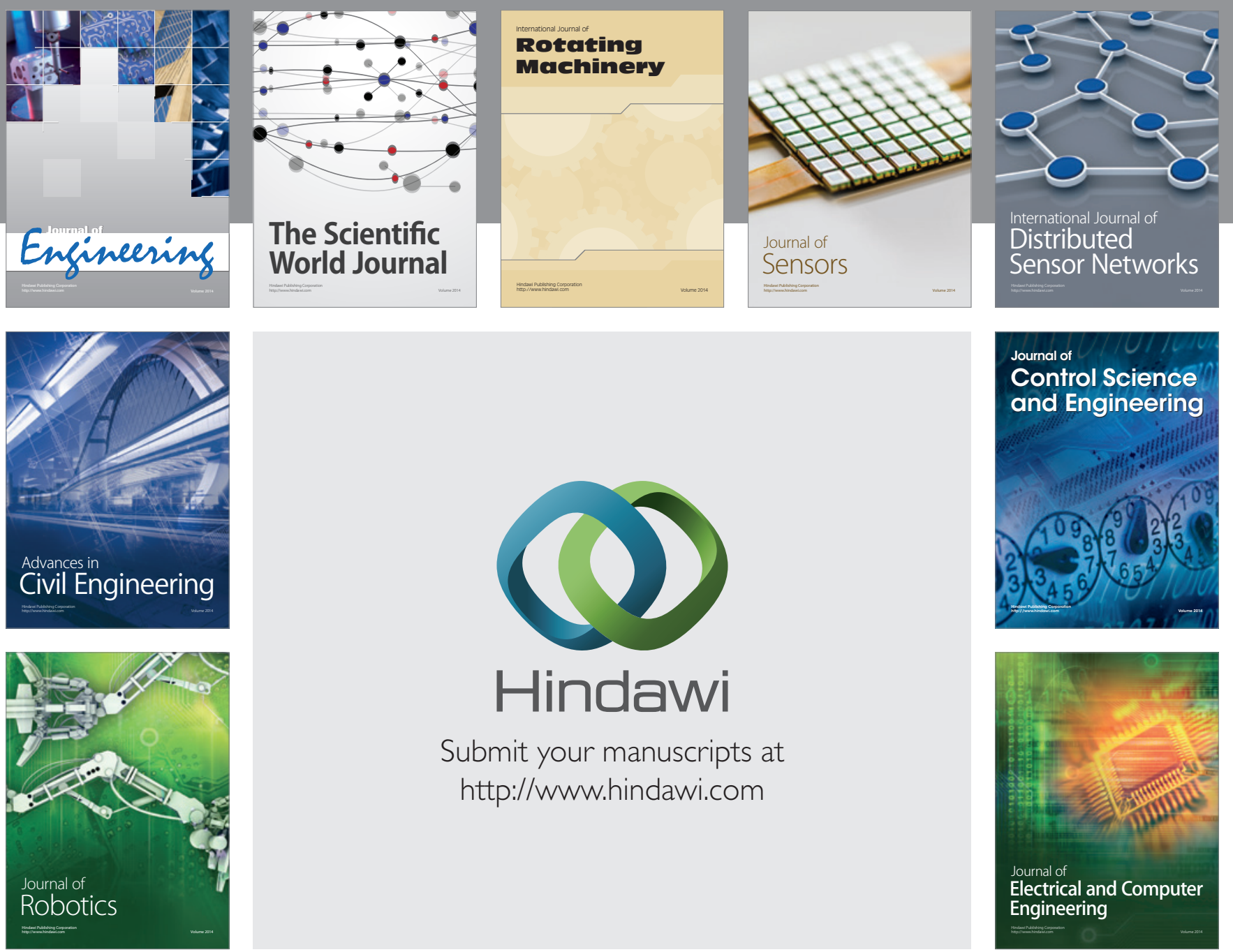

Submit your manuscripts at

http://www.hindawi.com
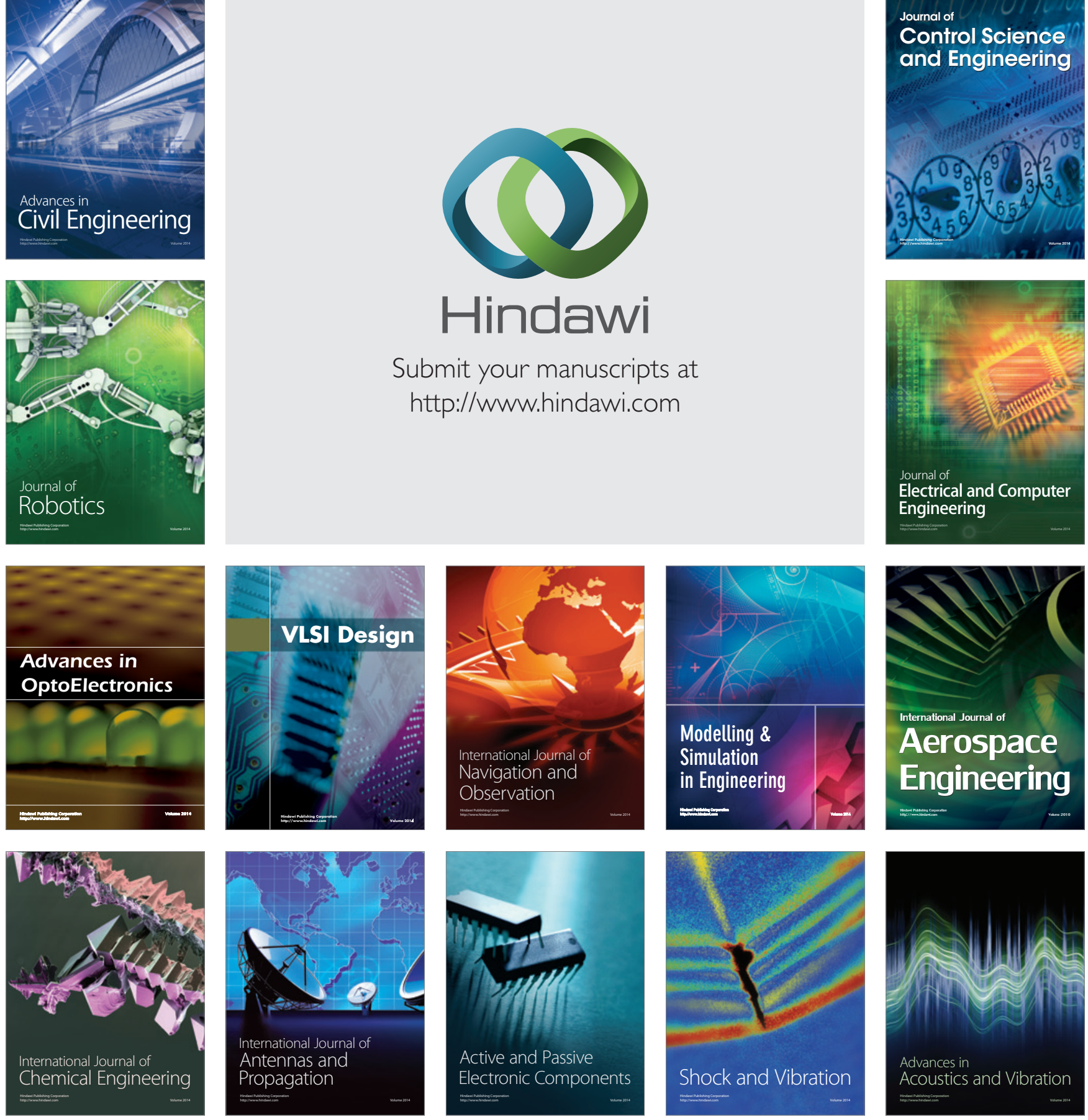